\title{
Image Enhancement Using Multi-objective Genetic Algorithms
}

\author{
Dinabandhu Bhandari, C.A. Murthy, and Sankar K. Pal \\ Center for Soft Computing Research, Indian Statistical Institute, \\ Kolkata 700108, India \\ dinabandhu.bhandari@gmail.com, \{murthy, sankar\}@isical.ac.in
}

\begin{abstract}
Given an image, there is no unique measure to quantitatively judge the quality of an image enhancement operator. It is also not clear which measure is to be used for the given image. The present work expresses the problem as a multi-objective optimization problem and a methodology has been proposed based on multi-objective genetic algorithm (MOGA). The methodology exploits the effectiveness of MOGA for searching global optimal solutions in selecting an appropriate image enhancement operator.
\end{abstract}

Keyword: Image Enhancement, Multi Objective Genetic Algorithms, Ambiguity Measures.

\section{Introduction}

There are many problems in the area of pattern recognition and image processing [1, where we need to perform efficient search in complex spaces in order to achieve an optimal solution. One such problem is contrast enhancement by gray-level modification, where the purpose is to improve the picture quality, more specifically, to improve the quality for visual judgment and/or machine understanding. In this case two major tasks are involved.

1. Selection of an appropriate transformation/mapping function (operator) for obtaining a desired output. Usually, a suitable nonlinear functional mapping is used to perform this task.

2. Selection of an evaluation function to define a quantitative measure of an enhanced image.

Not every kind of nonlinear function will produce a desired (meaningful) enhanced version [2]. Given an image, it is difficult to select a functional form which will be best suited without prior knowledge of the image statistics. Even if we are given the image statistics it is sometimes possible only to estimate approximately the function required for enhancement 23. The selection of the exact functional form still needs human interaction in an iterative process. To make process of evaluation of the quality of an image (picture) objective, it is necessary to define an objective function which will provide a quantitative 
measure for enhancement quality. Various evaluation functions are available in literature such as entropy, compactness, index of area coverage (IOAC), Divergence etc. to measure automatically the quality of the enhanced image. It has also been observed that all these measures are suitable for different kinds of images.

The effectiveness of multi-objective genetic algorithm (MOGA) is exploited to find a number of solutions in the Pareto-optimal front. Then depending on the image characteristics, an optimal solution is used as enhancement operator. Identifying the Pareto front is useful because it can be used to make well-informed decisions that balance trade-offs between the objectives. The problem here is to select automatically an optimum set of parameter values of 4 basic enhancement functions and their combining weightages that optimizes the fitness value (evaluation measure). The algorithm uses both spatial and grayness ambiguity measures to qualitatively evaluate the enhanced image. The methodology is demonstrated on a wide variety of images and obtained satisfactory results. Due to scarcity of space only a few have been presented in this paper. The problem of selecting an image enhancement operator and objective functions are described in section 2. Proposed methodology based on MOGA is explained in Section 3. Section 4 presents the results.

\section{Image Enhancement Operator and Objective Functions}

In the problem of gray-level re-scaling, each pixel is directly quantized to a new gray level in order to improve the contrast of an image. The simplest form of the functional mapping may be expressed as

$$
x_{m n}^{\prime}=x_{\max } \cdot f\left(x_{m n}\right)
$$

where, $x_{m n}=$ gray value of the $(m, n)$ th pixel of the input image (original), $x_{m n}^{\prime}=$ transformed value of the $(m, n)$ th pixel (enhanced), $x_{\max }=$ maximum value of the gray level dynamic range and $f(x)$ is the prescribed transformation function.

Most commonly used transformation functions [2,3/4] are shown in fig. 1. The mapping function $f_{1}($.$) depicted in Figure 1$ (a) increases the contrast within the darker area of the image, while the application of a function $f_{3}($.$) as in$ Figure 1 (c) will produce effects exactly opposite to that of function $f_{1}()$. The function $f_{2}($.$) shown in Figure 1$ (b) will result in stretching of the middle range gray levels and the function $f_{4}($.$) , in Figure 1$ (d), will drastically compress the middle range values, and at the same time it will stretch the gray levels of the upper and lower ends. The mathematical forms of the above mentioned mapping functions are given below.

$$
\begin{aligned}
f_{1}(x) & =\frac{A x^{2}}{1+A x^{2}} \quad=\frac{x^{2}}{\operatorname{par}_{1}+x^{2}} \quad \text { or } \\
& =\operatorname{par}_{1} \log (x)
\end{aligned}
$$

where, $\operatorname{par}_{1}$ and $A$ are positive constants.

$$
f_{2}(x)=\left[1+\left(\frac{x_{\max }-x_{\min }}{\operatorname{par}_{6}}\right)^{\text {ar }_{7}}\right]^{-1}
$$




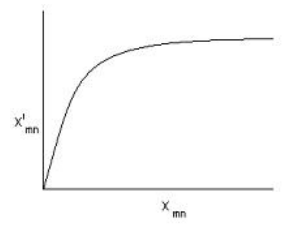

(a)

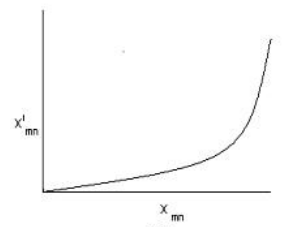

(c)

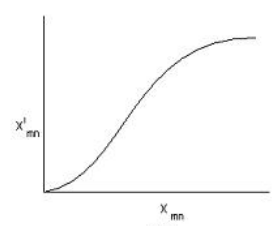

(b)

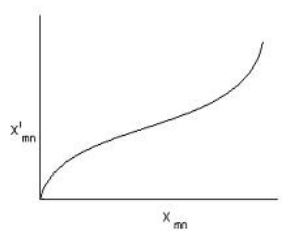

(d)

Fig. 1. Mapping functions commonly used for image enhancement

where, $\operatorname{par}_{6}$ and $\operatorname{par}_{7}$ are positive constants, and $x_{\min }$ and $x_{\max }$ are the minimum and maximum gray levels in the image.

$$
\begin{aligned}
f_{3}(x) & =\operatorname{par}_{2}[G(x)]^{2}+\operatorname{par}_{3} x+\operatorname{par}_{4} & & \text { where } 0<\operatorname{par}_{2}, \operatorname{par}_{3}, \operatorname{par}_{4}<1, \\
G(x) & =x-\operatorname{par}_{5} & & \text { for } x>\operatorname{par}_{5} \\
& =0 & & \text { Otherwise }
\end{aligned}
$$

where, $x_{\min }<\operatorname{par}_{5}<x_{\max }$. Finally, the function in Figure $2(\mathrm{~d})$ is given as:

$$
f_{4}(x)=\frac{1}{x_{\max }}\left[x_{\max }-\operatorname{par}_{6}\left\{\left(\frac{x_{\max }}{x}+\operatorname{par}_{8}\right)-1\right\}^{-\operatorname{par}_{7}}\right]
$$

where, $\operatorname{par}_{6}$ and $\operatorname{par}_{7}$ are positive constants, and $\operatorname{par}_{8}$ is the value of $f(x)$ for $x=0$.

Not all nonlinear functions will produce desired (meaningful) enhanced versions 2 of a particular image. Given an image, it is very difficult to decide a nonlinear functional form that will be best suited for highlighting its object without prior knowledge of image statistics. Even if the image statistics is known, it is possible only to estimate approximately the function required for enhancement and the selection of the exact functional form still needs human interaction in an iterative process. Since we do not know the exact function which will be suited for a given image, it seems appealing and convenient to use one general functional form which will yield the four functions mentioned above as special cases and possibly others. As an illustration one may consider a convex combination of these four functions e.g.,

$$
\begin{aligned}
f(.)= & \operatorname{par}_{9} f_{1}(.)+\operatorname{par}_{10} f_{2}(.)+\operatorname{par}_{11} f_{3}(.)+\operatorname{par}_{12} f_{4}(.) \text { where } \\
& \operatorname{par}_{9}+\operatorname{par}_{10}+\operatorname{par}_{11}+\operatorname{par}_{12}=1
\end{aligned}
$$

Here, the multipliers $\left(\operatorname{par}_{9}, \operatorname{par}_{10}, \operatorname{par}_{11}, \operatorname{par}_{12}\right)$ are to be chosen according to the importance (suitability) of a function for a given image. On the other hand, parameters $\left(\operatorname{par}_{1}, \operatorname{par}_{2}, \ldots\right.$, par $\left._{8}\right)$ of the respective functions, are to be defined 
according to the quality of enhancement desired. It may be noted that this combination will enable one to stretch/compress any region of an image one may desire. Therefore, the selection of a transformation function boils down to determining an optimum set of values of these 12 parameters in order to achieve a desired enhancement.

Once the enhancement function is known, to quantify the enhancement quality of the image individual judgment is required that makes the optimal decision subjective. Therefore, we need an evaluation function for quantifying the enhanced output, i.e., to objectively evaluate the subjective evaluation. One can consider entropy $(\mathrm{H})$, compactness, IOAC as they have been successfully used as grayness and spatial ambiguity measures for image enhancement and segmentation problems [56/7/4. Entropy of an image (X) considers the global information and provides an average amount of fuzziness in grayness of $\mathrm{X}$, i.e., the degree of difficulty (ambiguity) in deciding whether a pixel would be treated as black (dark) or white (bright). Compactness and IOAC on the other hand, take into account the local information and reflect the amount of fuzziness in shape and geometry (spatial domain) of an image. Therefore, the concept of minimization of these ambiguity measures may be considered as the basis of a fitness (evaluation) function. In 4], a composite measure (e.g., product of both grayness and spatial ambiguity measures) is used as the evaluation function so that minimization of this composite measure implies achieving minimum ambiguity (fuzziness) of an image. However, the composite measure may not be an ideal choice as each measure is given equal importance. For example, for an image consisting of a compact object, compactness would be given higher importance than IOAC. On the other hand, for an image consisting of an elongated object, IOAC would be given more importance. Instead this can be viewed as a multi-objective optimization problem. One can find the Pareto optimal solutions and finally, select the best transformation function based on the image characteristics.

\section{Proposed Methodology}

In this work, the real coded genetic algorithm is adapted, where a string of 12 real numbers is considered as chromosome. The domains of the parameters considered are shown in the following table.

\begin{tabular}{|l|c|}
\hline Parameters & Range \\
\hline par $_{1}-$ par $_{4}$ and par $_{8}-$ par $_{12}$ & {$[0,1]$} \\
par $_{5}$ and par $_{6}$ & {$\left[x_{\text {min }}, x_{\max }\right]$} \\
par $_{7}$ & {$[1,3]$.} \\
\hline
\end{tabular}

The single point crossover operation is adapted here with crossover probability 0.8 and mutation probability is varied from 0.3 to 0.01 with iteration. The concept of minimization of Entropy, Compactness and index of area coverage is considered as the basis of a fitness (evaluation) functions.

The non-dominated sorting algorithm NSGAII, proposed by Srinivas and Deb [89], is adopted in implementing the proposed methodology. The population size is assumed to be 100 . Once the child population is generated using the 
parent population, both the populations are combined together. Among these 200 offspring and parent solutions, 100 are being selected for the selection, crossover and mutation operations and a child population of same size is created. The non-dominated sorting is then used to classify the entire population. Once the non-dominated sorting is over, the new population of size 100 is generated taking solutions from the best non-dominated front and continues with second front and so on. The algorithm ensures the diversity among the selected solution in the parent population. However, during the later stages of the algorithm, it is likely that most solutions in the population lie in the best non-dominated front.

\section{Implementation and Results}

The proposed methodology has been tested on several images, having bimodal as well as multi-modal histograms. The images consist of compact as well as elongated objects. The algorithm uses the standard $S$-function of Zadeh to calculate the fuzziness of a pixel. Three measures entropy, compactness and IOAC of the images are used as the evaluation functions. The algorithm is executed for 500 iterations. The Pareto front obtained after 500 iterations contains 100 solutions. Then depending on the image characteristics, a solution set is taken to transform the image. For the simulation, gray scale [0, 255] images of size $256 \times 256$ pixels are used. In order to evaluate the performance of the proposed methodology, results are compared with the methodology developed using simple genetic algorithm (SGA) with elitist model. In the implementation of SGA, composite evaluation measure (product of entropy, compactness and IOAC) is minimized to obtain the optimum solution. The algorithm is also executed 500 iterations with a population size 100 .

Fig 2(a) illustrates a low contrasted image. 2(b) and 2(c) depict the output obtained using SGA and MOGA based methodologies. Out of the several solutions obtained in the Pareto front using MOGA, the solution producing medium entropy, and low compactness and IOAC measures are found to produce better results for enhancement. This may be due to the reason that entropy tends to 0 when there is least ambiguity that means all the pixels lie either in object or background. Similar results are also found for the image provided in Fig 3(a).

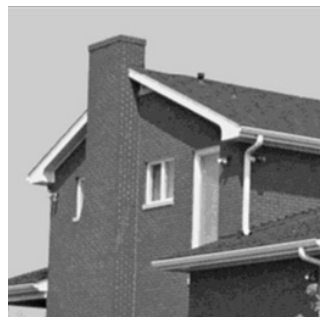

(a)

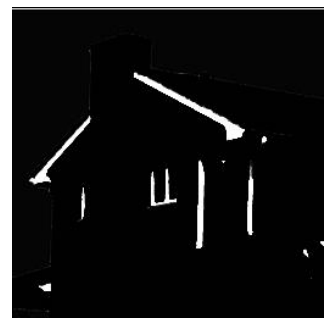

(b)

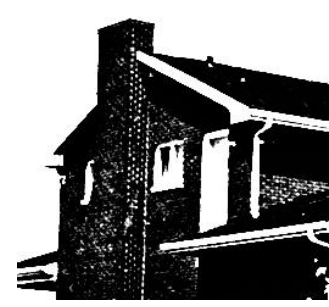

(c)

Fig. 2. The House image (a) (b) and (c) 


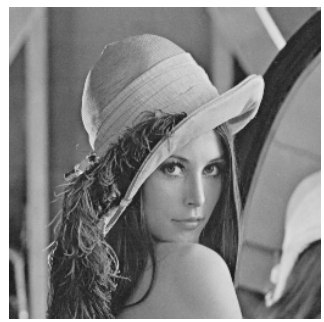

(a)

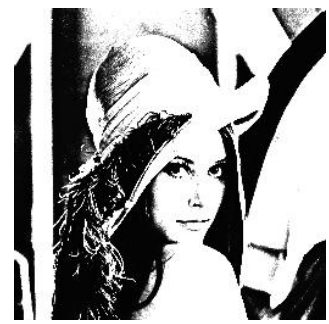

(b)

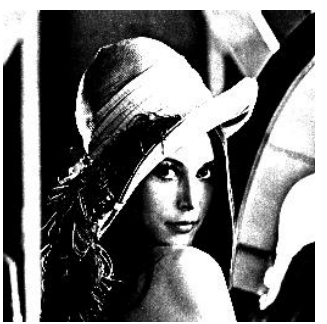

(c)

Fig. 3. Leena image (a) (b) and (c)

Comparing the images of figs 2 and 3, one can observe the efficiency of the proposed method in enhancing the images.

\section{Conclusions}

The effectiveness of MOGA in the automatic selection of an optimum image enhancement operator is tested for various bimodal and multimodal images. Although fuzziness measures have been used as fitness values, one may use other measures depending on the problems. Moreover, one can use more measures as objective functions. The algorithm determines number of optimum parameter sets rather than a single set in selecting an appropriate enhancement function.

\section{References}

1. Rosenfeld, A., Kak, A.C.: Digital picture processing. Academic Press, New York (1982)

2. Ekstrom, M.P.: Digital image processing techniques. Academic Press, New York (1984)

3. Kundu, M.K., Pal, S.K.: Automatic selection of object enhancement operator with quantitative justification based on fuzzy set theoretic measure. Pattern Recognition Letters 11, 811-829 (1990)

4. Pal, S.K., Bhandari, D., Kundu, M.K.: Genetic algorithms for optimal image enhancement. Pattern Recognition Letters 15, 261-271 (1994)

5. Vlachos, I.K., Sergiadis, G.D.: Parametric indices of fuzziness for automated image enhancement. Fuzzy Sets and Systems 157, 1126-1138 (2006)

6. Cheng, H.D., Li, J.: Fuzzy homogeneity and scale-space approach to color image segmentation. Pattern Recognition 36, 1545-1562 (2003)

7. Munteanu, C., Rosa, A.: Gray-scale image enhancement as an automatic process driven by evolution. IEEE Transactions on Systems, Man and Cybernetics-Part B: Cybernetics 34(2), 1292-1298 (2004)

8. Srinivas, N., Deb, K.: Multiobjective function optimization using nondominated sorting genetic algorithms. Evolutionary Computation Journal 2(3), 221-248 (1995)

9. Deb, K.: Multi-Objective Optimization Using Evolutionary Algorithms. John Wiley, Chichester (2001) 